\title{
Comparativa entre islas de la valoración de distintos tipos de oferta turística por parte de los residentes: el caso de las Islas Baleares (España)
}

\section{Comparison between islands in the valuation of different types of tourism by residents: the case of the Balearic Islands (Spain)}

\author{
Jose Ramón Cardona (RAMÓN CARDONA, J.)
}

\begin{abstract}
RESUMEN - El turismo depende de la buena voluntad de los residentes, siendo fundamental el apoyo de la población local al desarrollo del sector. Los tipos y formas de turismo influyen en cómo evolucionan las actitudes de los residentes. Pero la inmensa mayoría de los estudios de actitudes de los residentes analizan el sector turístico en su conjunto, siendo pocos los casos que se centran en algún tipo concreto de oferta. El objetivo de este trabajo era analizar si había productos turísticos con peor o mejor valoración por parte de los residentes de las Islas Baleares. Se realizó una comparación por islas y una segmentación de los residentes según su valoración de los tipos de producto turístico. Las ofertas turísticas que generaban menor impacto en la comunidad residente e implican un mayor interés de los turistas por la sociedad y el entorno local poseían mayor grado de aceptación, mientras que la oferta turística con más impactos negativos y que implica menor interacción con los residentes poseía bajos niveles de aceptación.
\end{abstract}

Palabras Clave: Baleares; Actitudes; Residentes; Producto turístico; Comparación.

ABSTRACT - Tourism depends on the goodwill of the residents, being essential the support of the local population to the development of the sector. The types and forms of tourism affect how evolving attitudes of the residents. But the immense majority of studies of residents' attitudes analyze the tourism sector as a whole, being few cases that focus on a particular type of supply. The objective of this work was to analyze if there was tourist products with worse or better rating by the residents of the Balearic Islands. A comparison is performed by islands and a segmentation of residents by assessing the types of tourism product. The tourist supply that generated less impact on the resident community and involve a greater interest of tourists by the society and the local environment had highest degree of acceptance, while the tourist supply with more negative impacts and that means less interaction with the residents had low levels of acceptance.

Key words: Balearic Islands; Attitudes; Residents; Tourism product; Comparison.

\footnotetext{
* Licenciado en Administración y Dirección de Empresas (Universitat de les Illes Balears), Licenciado en Economía (Universitat de les Illes Balears) y Doctorado en Economía de la Empresa (Universitat de les Illes Balears). Investigador colaborador del grupo de Dirección y Gestión de Empresas y Destinos Turísticos (Universitat de les Illes Balears). Dirección: Casa Can Pilot (Buscastell), no 15.047. C.P. 07820 - Sant Antoni de Portmany (Illes Balears) - España. Teléfono: (+34) 971343789 / 6968085 32. E-mail: jramon.cardona@terra.com
} 


\section{INTRODUCCIÓN}

El turismo depende de la buena voluntad de los residentes, siendo fundamental el apoyo de la población local al desarrollo del sector (BESCULIDES; LEE; MCCORMICK, 2002; GURSOY; JUROWSKI; UYSAL, 2002). Además se encuentra ampliamente reconocido que los gestores deben tener en cuenta los puntos de vista de los residentes, si se desea la sostenibilidad del turismo (AP; CROMPTON, 1998; MADDOX, 1985), y a lo largo de las últimas décadas se han realizado diversos estudios analizando las actitudes hacia el turismo (BESCULIDES; LEE; MCCORMICK, 2002; FREDLINE; FAULKNER, 2000; GURSOY; KENDALL, 2006; GURSOY; RUTHERFORD, 2004; HARALAMBOPOULOS; PIZAM, 1996; JUROWSKI; GURSOY, 2004; JUROWSKI; UYSAL; WILLIAMS, 1997; KIM; PETRICK, 2005; MASON; CHEYNE, 2000; TEYE; SIRAKAYA; SÖNMEZ, 2002; WEAVER; LAWTON, 2001; WILLIAMS; LAWSON, 2001).

Los tipos y formas de turismo y, sobre todo, la percepción y actitud de los turistas influyen en cómo evolucionan las actitudes de los residentes con el paso del tiempo una vez iniciada la actividad turística (MURPHY, 1985; RITCHIE, 1988; WILLIAMS; LAWSON, 2001), y su importancia es tan grande que las actitudes y percepciones de los turistas y de los residentes deben estudiarse como elementos interrelacionados y no de forma separada. Pero la inmensa mayoría de los estudios de actitudes de los residentes analizan el sector turístico en su conjunto, siendo pocos los casos que se centran en algún tipo concreto de turismo o actividad. La única excepción se produce en los destinos con una elevada especialización en el juego y los casinos (BROWN; ROSEMAN; HAM, 2003; JANES; COLLISON, 2004; LEE; KIM; KANG, 2003; VONG, 2009; VONG; MCCARTNEY, 2005).

El objetivo de este trabajo era analizar si había productos turísticos con peor o mejor valoración por parte de los residentes de las Islas Baleares, mediante un estudio descriptivo que medía la valoración de los diversos productos turísticos, comparaba Mallorca, Menorca e Ibiza y segmentaba los residentes según su valoración general de estas ofertas.

Las Islas Baleares es un archipiélago del Mediterráneo occidental compuesto por cuatro islas habitadas y diversas islas e islotes deshabitados (Figura 1). El archipiélago 
posee una superficie de 4.984,6 $\mathrm{km}^{2}$ repartido entre Mallorca $\left(3.622,5 \mathrm{~km}^{2}\right.$ ), Menorca $\left(694,7 \mathrm{~km}^{2}\right)$, Ibiza $\left(571,8 \mathrm{~km}^{2}\right)$, Formentera $\left(82,5 \mathrm{~km}^{2}\right)$, Cabrera $\left(13,0 \mathrm{~km}^{2}\right)$ y diversos islotes, según datos del Institut Balear d'Estadística (IBESTAT, 2012). La población residente a 1 de enero de 2012 era de 1.119.439 habitantes repartido entre Mallorca (876.147 habitantes), Menorca (95.178 habitantes), Ibiza (137.357 habitantes) y Formentera (10.757 habitantes) (IBESTAT, 2012).

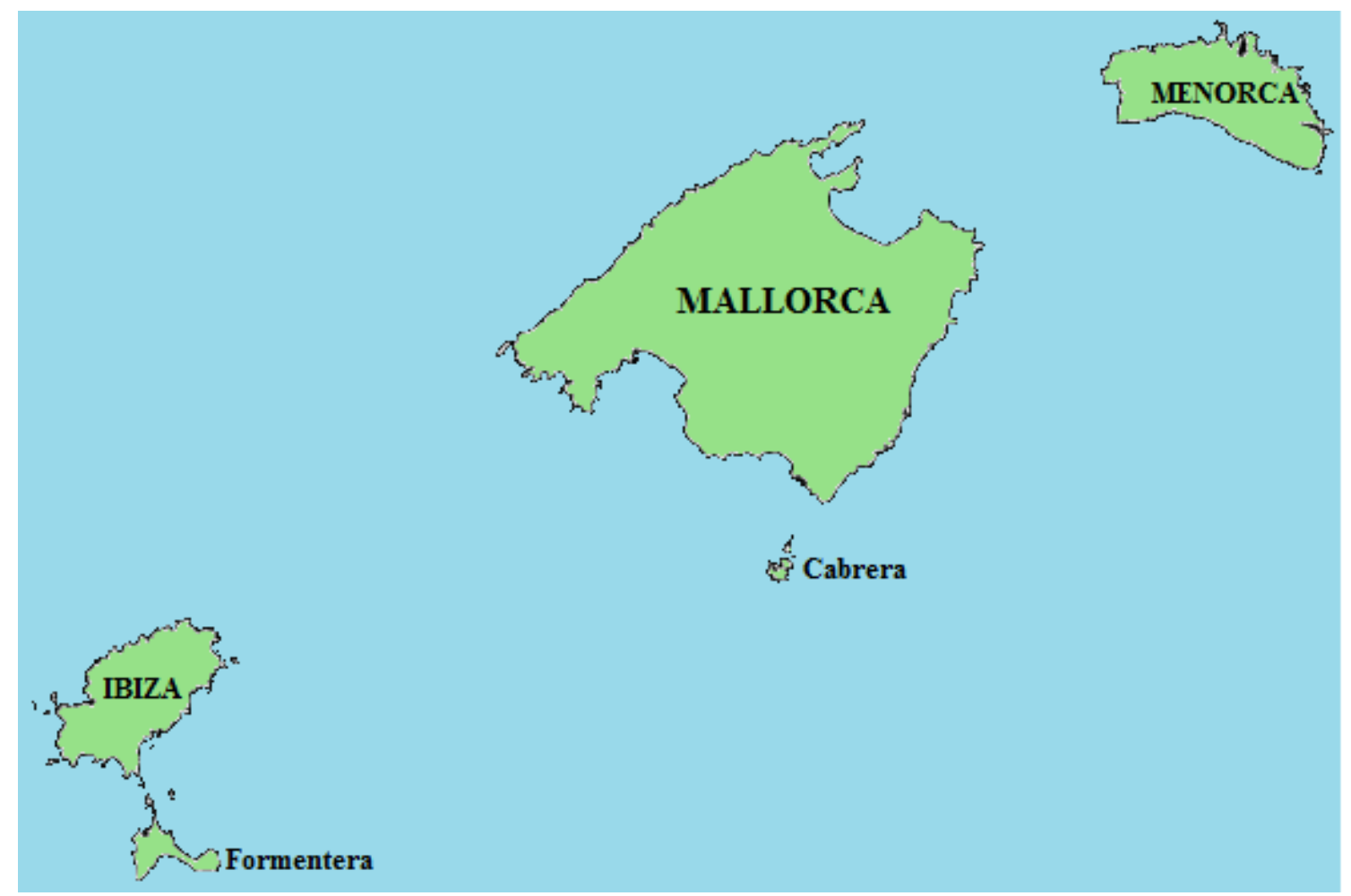

FIGURA 1 - MAPA DE LAS ISLAS BALEARES.

Fuente: Elaboración propia.

En el año 2012, Baleares recibió 12.561.515 turistas que generaron 105.810.989 pernoctaciones (AGÈNCIA DE TURISME DE LES ILLES BALEARS, 2013). Por islas, Mallorca recibió 9.146.966 turistas, principalmente alemanes (3.450.345 turistas), británicos (1.985.561 turistas) y españoles (1.189.995 turistas); Ibiza y Formentera recibieron 2.313.104 turistas, destacando británicos (702.671 turistas) y españoles (630.443 turistas) seguidos por italianos (300.980 turistas) y alemanes (275.458 turistas); y Menorca recibió 1.101.446 turistas, principalmente españoles (434.547 turistas) y británicos (415.305 turistas) (AGÈNCIA DE TURISME DE LES ILLES BALEARS, 2013). El gasto realizado por los turistas se estimó en 11.456.396.554 € en 2012, con un gasto medio diario de $108,3 €$ (destacando Ibiza y Formentera con un 
gasto medio diario de 121,1 €) (AGÈNCIA DE TURISME DE LES ILLES BALEARS, 2013). Las Islas Baleares poseen una fuerte dependencia económica del turismo y se encuentran tradicionalmente especializadas en el turismo de sol y playa familiar, aunque con algunas diferencias: Ibiza es conocida por el ocio nocturno y Menorca por el grado de conservación de su naturaleza.

\section{MARCO TEÓRICO: ACTITUDES Y TIPOS DE TURISMO}

Para la sostenibilidad de los destinos turísticos es importante conocer las actitudes y opiniones de los residentes hacia el turismo, y es esperable que estas actitudes varíen según el tipo de turismo existente. A la hora de estudiar los tipos de turistas la clasificación debe realizarse según sus percepciones y actitudes en el destino turístico más que por cualquier otro elemento de clasificación, ya que eso es lo que afecta las actitudes de los residentes. No es lo mismo un turista que muestra respeto e interés por la cultura y la sociedad local que un turista que sólo busca libertad a bajo precio y en un ambiente similar a su lugar de origen. En relación al turismo mediterráneo, se puede dibujar el perfil de cuatro tipos de turistas (LANQUAR, 2001):

- El "Sedentario". Sus principales motivaciones para el viaje son el sol, el mar y la playa, no le apetece apartarse de sus hábitos y realiza vida diurna, frecuenta establecimientos de categoría media, le gustan las fotos y los recuerdos, y posee afición por las excursiones. Son personas sin gran curiosidad intelectual y que viajan en verano.

- El “Sedentario-Móvil”. Le gusta el deporte, visitar lugares y monumentos y realizar fotografías. Realizan estancias vacacionales de más de una semana, para rentabilizar la distancia recorrida.

- El "Itinerante" es una persona que busca un cambio cultural y social, y que pertenece a la élite económica o economiza para viajar. Le gusta visitar todo lo que aparece en las guías, realizar fotografías y actividades folclóricas, adquirir recuerdos exóticos, comer comida internacional y probar la comida local. Es un tipo de turista poco numeroso y que realiza viajes de dos o tres semanas de duración.

- El "Nómada" es una persona que busca el verdadero contacto con la población local y la naturaleza. Está muy motivado (las motivaciones son muy variadas), posee un 
buen nivel educativo y es joven. Realiza el viaje de forma individual o en pequeños grupos, está abierto a experiencias culinarias, se aloja en hoteles locales y busca el folclore auténtico.

Otra tipología psicográfica de los turistas es la propuesta por Bigné, Font y Andreu (2000):

- Los "extremos impulsores" viajan por motivos educativos o culturales, búsqueda de lo exótico y lo nuevo, encontrar amistades de otras culturas, búsqueda de libertad e independencia, etc.

- Los “de intereses especiales" se desplazan por motivos religiosos, asistir a competiciones deportivas o espectáculos especiales, realizar viajes de aventura, etc.

- Los “intermedios" buscan el descanso y relax, la salud, el cambio de vida, el encanto del lugar, la naturaleza, el lujo y el romance, las compras, etc.

- Los "extremos conformistas" desean ambiente familiar, posición social, viajes a lugares conocidos, etc.

Según los tipos de destino elegidos por los turistas, Plog (1974) estableció una tipología con los alocéntricos (buscan lugares desconocidos sin desarrollo turístico) y los psicocéntricos (huyen de lugares desconocidos y visitan destinos consolidados) en los extremos. Entre ambos se sitúan los céntricos como grupo mayoritario (Figura 2). Los psicocéntricos son habituales en destinos consolidados como Baleares.

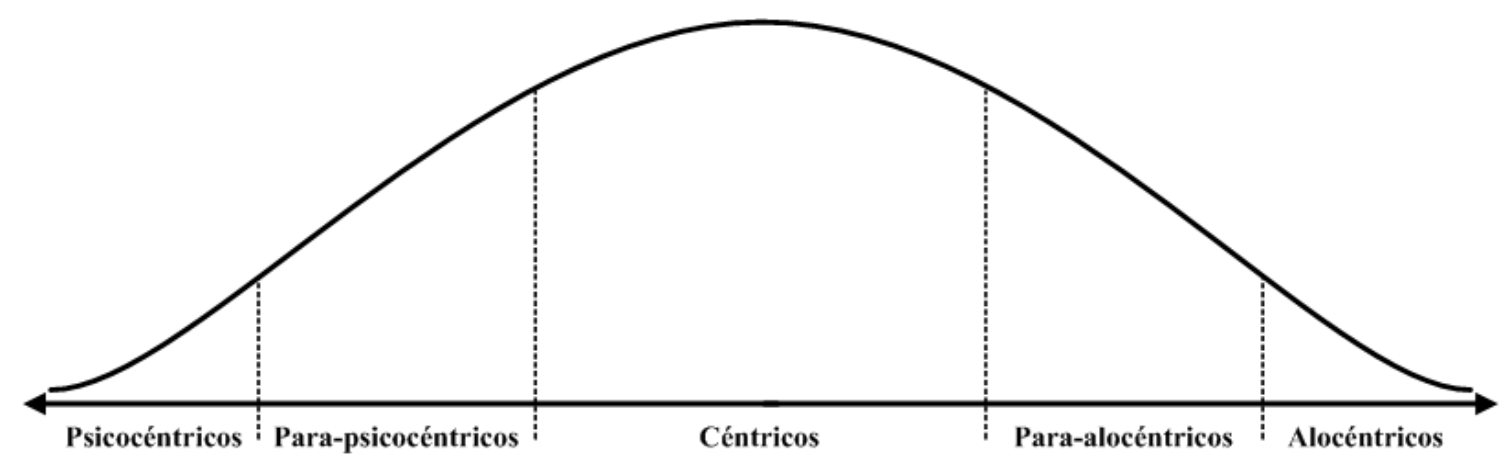
FIGURA 2 - TIPOLOGÍAS DE TURISTAS.

Fuente: Plog (1974).

Los turistas con motivaciones culturales y aventureras (nómadas, extremos impulsores, alocéntricos, etc.) suelen mostrar mayor respeto por la población residente y suelen realizar un mayor índice de actividades durante su estancia (PLOG, 1974). Pueden llegar a ser molestos por excesivamente curiosos pero suelen tener una imagen 
del destino buena, y eso ayuda a que los residentes tengan mejor actitud hacia los turistas y ofrezcan mayor grado de hospitalidad. Otro tipo de turismo que puede influir positivamente en la actitud de los residentes son los poco activos, es decir los que buscan tranquilidad y descanso.

En el otro extremo pueden encontrarse los turistas sin ningún interés cultural por la región, que no buscan la tranquilidad, más bien huyen de ella y consideran que el destino turístico, residentes incluidos, es quien debe adaptarse a los turistas y no viceversa (sedentarios, extremos conformistas, psicocéntricos, etc.) (PLOG, 1974). Este tipo de turistas suelen causar más perjuicios y los principales afectados son los residentes de las zonas donde se concentran.

\subsection{ACTITUDES DE LOS TURISTAS}

En muchos casos las actitudes de los turistas hacia el destino cambian una vez finalizada la estancia a causa del formato de viaje y las actitudes previas. El contacto cultural entre turistas y residentes es importante para cambiar las actitudes de los turistas hacia el destino turístico a través de un aumento del nivel de satisfacción que obtienen con la estancia en el destino. Gómez, San Martín y Bertiche (1999) propusieron un modelo en donde la interacción intercultural (turistas con residentes, principalmente), las actividades turísticas realizadas y la calidad de los servicios recibidos afectan de forma indirecta sobre las actitudes y los estereotipos de los turistas hacia el destino a través de la satisfacción con la estancia. En su estudio se diferenciaron tres tipos de motivaciones para realizar el viaje: culturales (conocimiento de otras culturas y sus diferencias con respecto a la del lugar de origen del turista), atractivo del destino (pull) y necesidades psicosociales del individuo que realiza el viaje (push) que le animan a alejarse de su rutina diaria.

Entre los resultados que obtuvieron se puede mencionar que los turistas que viajan por motivos culturales realizan más actividades en el destino turístico (con un mayor contacto con la población autóctona). Los turistas que se desplazan por motivaciones psicosociales suelen interactuar de forma más fuerte que los demás con la población local. La interacción con la población local es un aspecto vital para la 
experiencia del turista (GÓMEZ, SAN MARTÍN; BERTICHE, 1999), y por tanto un elemento fundamental en la prosperidad de la "industria turística".

Una característica de algunos turistas es, curiosamente, la presencia de actitudes antiturísticas (BRUCKNER; FINKIELKRAUT, 1979, p. 37-41; JACOBSEN, 2000). Los turistas con actitudes antiturísticas no quieren mezclarse con los turistas, es decir quieren vivir una experiencia original y no preparada para ser vivida por una masa de turistas, ya que no se consideran turistas (FISCHER, 1984; HENNIG, 1997). Las expectativas de estos individuos (viajeros) son diferentes de las de los individuos que no muestran este tipo de actitudes (turistas). En el caso de los viajeros se percibe una tendencia a experimentar la comida local y a explorar los lugares por ellos mismos (PEARCE, 1982). Por contra el turismo tradicional aporta protección en las experiencias en el extranjero y “evita” la involucración de los turistas en la cultura local. Como argumenta Scheuch (1972), el turismo organizado ha sido desarrollado para evitar el problema del cambio de lugar y la inadaptación inicial que se sufriría si se viajara de forma independiente. Los individuos con actitudes antiturísticas adoptan una posición de distancia al realizar viajes $\mathrm{y}$, aunque realicen viajes organizados, buscan permanecer separados y distintos del que se considera el típico turista (JACOBSEN, 2000), no se consideran turistas conformistas y no quieren que se les identifique como tales (GOFFMAN, 1961).

Las actitudes antiturísticas son, en parte, una crítica del turismo organizado y los paquetes turísticos (JACOBSEN, 2000) y se encuentran relacionadas con la idea de esperar una superficialidad de la experiencia que aporta el turismo tradicional, especialmente en el turismo de grupos organizados (URBAIN, 1991).

Las actitudes antiturísticas se han convertido en un símbolo de prestigio ya que con ellas se busca no ser considerado un turista ordinario (BRUCKNER; FINKIELKRAUT, 1979). Estas actitudes son una acentuación del individualismo y tiene elementos en común con los turistas alocéntricos (PLOG, 1974).

\subsection{INTERACCIÓN ENTRE LOS RESIDENTES Y LOS TURISTAS}

El tipo e intensidad de los contactos entre visitantes y residentes han sido consideradas variables importantes en la determinación de las actitudes de los residentes 
(PEARCE, 1996), sobre todo cuando el residente trabaja o depende económicamente del turismo (MILMAN; PIZAM, 1988). La interacción entre la población residente y los visitantes es uno de los factores más importante tanto para determinar las percepciones y actitudes de los residentes (MURPHY, 1985), como para determinar los niveles de satisfacción de los turistas a través de la percepción que obtienen del destino turístico (GÓMEZ; SAN MARTÍN; BERTICHE, 1999).

Las actitudes de los turistas cuando llegan a la región determinan las acciones que realizan. Si tienen una actitud de respeto hacia los elementos locales sus acciones son respetuosas dentro de los conocimientos de que disponen. Las actitudes de los residentes también son expresadas a través de sus acciones, principalmente la hospitalidad ofrecida a los turistas. Las acciones de los residentes se convierten en uno de los elementos que determinan con mayor fuerza la percepción que tienen los turistas del viaje, provocando de esta forma que las actitudes de los residentes se transmitan a los turistas mejorando o empeorando la imagen que tienen del destino. Por tanto se forma un circuito en donde los residentes y los turistas se influyen mutuamente (Figura 4). La evolución de este circuito marca enormemente la calidad de vida de los residentes (molestias o beneficios que sufren) y el nivel de hospitalidad que reciben los turistas y que determina el nivel de satisfacción con su viaje.

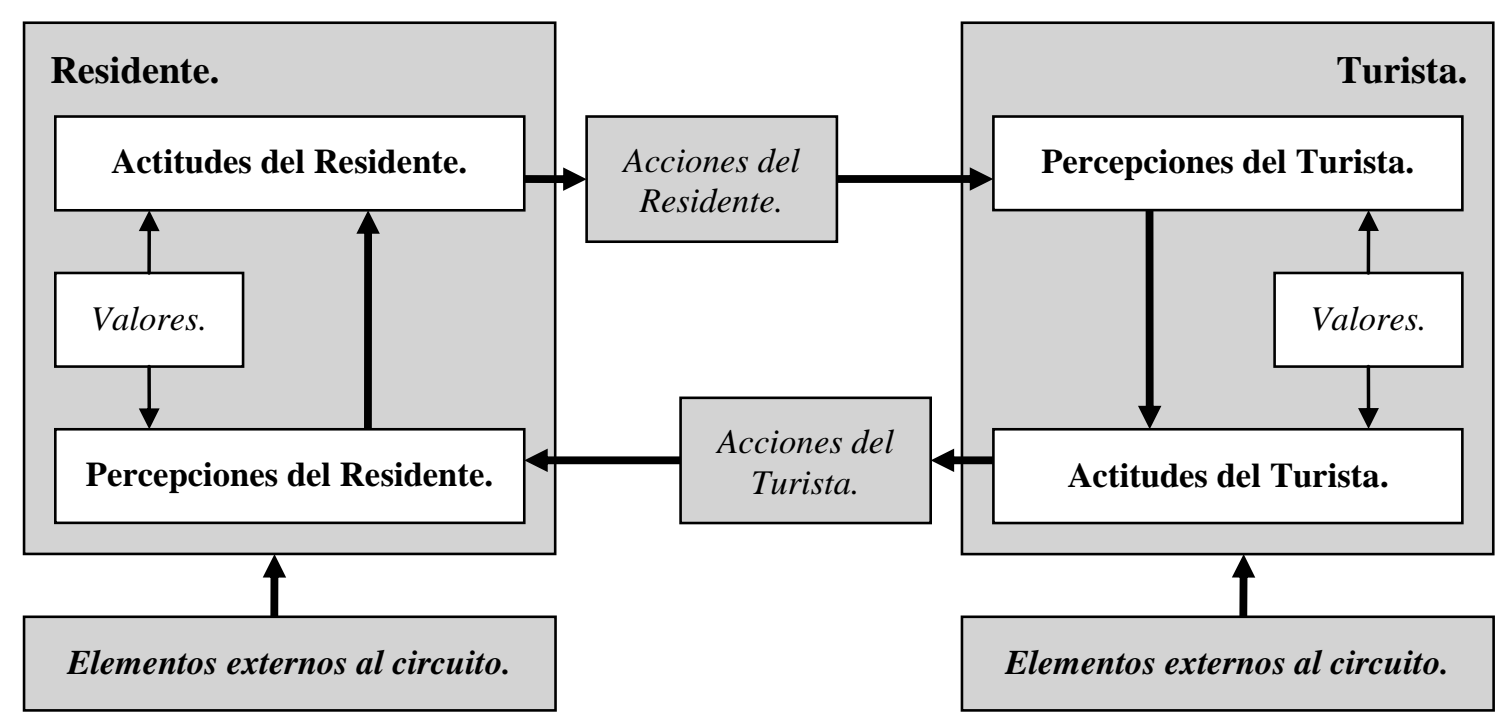

FIGURA 4 - CIRCUITO DE INTERRELACIONES ENTRE RESIDENTES Y TURISTAS.

Fuente: elaboración propia. 


\section{METODOLOGÍA}

El análisis de las actitudes de los residentes hacia diversos productos u ofertas turísticas permite determinar el desarrollo turístico más adecuado para el destino, consiguiendo mayor aceptación del sector turístico por parte de los residentes y mayor hospitalidad hacia los turistas. Redundando todo ello en una mayor sostenibilidad del sector en el largo plazo y una mayor implicación social en la gestión turística. En este caso se analizó la valoración de diversos productos turísticos por parte de los residentes de las Islas Baleares, realizando una comparación de las distintas ofertas y de las tres islas principales (Mallorca, Menorca e Ibiza).

Para el análisis se usó una encuesta con muestreo aleatorio y entrevista personal. La valoración de los productos turísticos se realizó mediante una escala Likert de 5 puntos, donde 1 es "Muy Inaceptable" y 5 es "Muy Aceptable". El trabajo de campo fue realizado en las tres islas principales (Mallorca, Menorca e Ibiza), obteniendo una muestra con 1.873 entrevistas realizadas en diversos momentos del año 2012 y con las características demográficas expuestas en la Cuadro 1. Un estudio realizado en Ibiza en 2011 con similares preguntas mostro misma prelación de las ofertas y la única diferencia era una puntuación ligeramente superior en todas las ofertas, posiblemente debido a la crisis económica iniciada en 2008. Por tanto, la ordenación y comparación de los tipos de oferta parece totalmente válida para la actualidad.

CUADRO 1 - CARACTERÍSTICAS DEMOGRÁFICAS DE LA MUESTRA.

\begin{tabular}{|c|c|c|c|c|}
\hline & $\begin{array}{l}\text { Baleares } \\
n=1873\end{array}$ & $\begin{array}{c}\text { Ibiza } \\
n=226\end{array}$ & $\begin{array}{l}\text { Mallorca } \\
\mathrm{n}=\mathbf{1 5 1 1}\end{array}$ & $\begin{array}{c}\text { Menorca } \\
\mathbf{n}=136\end{array}$ \\
\hline \multicolumn{5}{|l|}{ SEXO } \\
\hline Hombre & $45,74 \%$ & $40,27 \%$ & $47,71 \%$ & $33,09 \%$ \\
\hline Mujer & $54,26 \%$ & $59,73 \%$ & $52,29 \%$ & $66,91 \%$ \\
\hline \multicolumn{5}{|l|}{ EDAD } \\
\hline Menos de 30 años & $43,22 \%$ & $26,11 \%$ & $45,30 \%$ & $48,53 \%$ \\
\hline entre 30 y 45 años & $29,49 \%$ & $34,51 \%$ & $28,41 \%$ & $33,09 \%$ \\
\hline entre 46 y 60 años & $20,09 \%$ & $26,55 \%$ & $19,60 \%$ & $14,71 \%$ \\
\hline Más de 60 años & $7,21 \%$ & $12,83 \%$ & $6,69 \%$ & $3,68 \%$ \\
\hline \multicolumn{5}{|l|}{ EDUCACIÓN } \\
\hline Sin estudios & $3,73 \%$ & $6,73 \%$ & $3,07 \%$ & $6,20 \%$ \\
\hline Estudios Primarios & $22,40 \%$ & $35,43 \%$ & $20,59 \%$ & $20,93 \%$ \\
\hline Estudios Secundarios & $37,23 \%$ & $36,32 \%$ & $36,50 \%$ & $47,29 \%$ \\
\hline Estudios Universitarios & $36,63 \%$ & $21,52 \%$ & $39,84 \%$ & $25,58 \%$ \\
\hline \multicolumn{5}{|c|}{ TRABAJA EN TURISMO } \\
\hline Trabaja en turismo & $40,03 \%$ & $53,85 \%$ & $35,61 \%$ & $67,18 \%$ \\
\hline No trabaja en turismo & $59,97 \%$ & $46,15 \%$ & $64,39 \%$ & $32,82 \%$ \\
\hline
\end{tabular}

Fuente: elaboración propia. 
Con los datos obtenidos se realizó una ordenación de los distintos tipos de oferta según las preferencias de los residentes y se analizaron las diferencias entre las islas con la ayuda de tablas ANOVA. El análisis se complementó con una segmentación de los residentes encuestados según sus valoraciones de los distintos tipos de oferta turística, mediante un análisis clúster, técnica con antecedentes de uso en la bibliografía (AGUILÓ; ROSSELLÓ, 2005; BRIDA; OSTI; BARQUET, 2010; FREDLINE; FAULKNER, 2000; WEAVER; LAWTON, 2001; WILLIAMS; LAWSON, 2001).

\section{VALORACIÓN DE DIVERSOS PRODUCTOS TURÍSTICOS}

En el estudio realizado se obtuvo como resultado general que eran considerados aceptables la mayoría de los productos, aunque unos más que otros (Cuadro 2). Si se analizaban los diversos productos uno por uno podía observarse que:

- El turismo rural surgió como parte de un binomio que debía servir como puntal de la actividad agraria del continente europeo, más allá del proteccionismo y las subvenciones. El objetivo era que la combinación de las denominaciones de calidad y el turismo rural permitiera que siguiera siendo rentable el trabajo agrario. La peculiaridad del turismo rural de Baleares era el nivel de calidad de la oferta. Mientras en otras partes de España y Europa los establecimientos rurales son llevados en muchos casos por la familia propietaria de la explotación y tienen como finalidad económica completar las rentas de la explotación, en Baleares eran muchos los establecimientos gestionados por personas con amplia experiencia hotelera y el personal estaba altamente cualificado. Era por tanto un turismo rural distinto, más profesionalizado de lo habitual y con una calidad propia de un hotel de cuatro o cinco estrellas a pequeña escala. Era el producto turístico mejor valorado en el conjunto de las islas $(4,2766)$ y destacaba la elevada aceptación que recibía por parte de los menorquines $(4,4559)$. El hecho de explotar un atractivo tradicionalmente no suficientemente explotado y la calidad de esta oferta explicaría esta elevada valoración.

- El turismo basado en la naturaleza (rutas excursionistas, zonas de observación de fauna, etc.) era uno de los más valorados (4,2472), junto al turismo rural, destacando la elevada valoración hecha por los residentes de Menorca $(4,4559)$. Se percibía un 
deseo entre los residentes de que se potenciaran atractivos que consideraban infrautilizados y uno de ellos era el paisaje natural. Además, el turismo consistente en la contemplación del entorno natural gozaba de buena imagen ya que llevaba implícito el respeto por el lugar visitado.

CUADRO 2 - COMPARATIVA DE LOS VALORES PARA LAS TRES ISLAS.

\begin{tabular}{lccccc}
\hline Producto Turístico & Baleares & Ibiza & Mallorca & Menorca & \\
n= 1873 & $\mathbf{n = 2 2 6}$ & $\mathbf{n = 1 5 1 1}$ & $\mathbf{n = 1 3 6}$ & \\
\hline Turismo Rural & 4,2766 & 4,3805 & 4,2449 & 4,4559 & $(*)$ \\
Turísticos de naturaleza & 4,2472 & 4,4159 & 4,2032 & 4,4559 & $(*)$ \\
Turismo cultura & 4,1570 & 4,3673 & 4,1125 & 4,3015 & $(*)$ \\
Congresos, Seminarios, Ferias & 4,0945 & 4,1726 & 4,0841 & 4,0809 & \\
Otro turismo deportivo & 3,9509 & 4,1858 & 3,9107 & 4,0074 & $(*)$ \\
Turismo de sol y playa familiar & 3,7058 & 4,1416 & 3,6334 & 3,7868 & $(*)$ \\
Cicloturismo & 3,6188 & 3,9867 & 3,5586 & 3,6765 & $(*)$ \\
Turismo Náutico & 3,5718 & 4,1106 & 3,4540 & 3,9853 & $(*)$ \\
Casas vacacionales en alquiler & 3,5195 & 3,8894 & 3,4242 & 3,9632 & $(*)$ \\
Golf & 3,3182 & 3,4602 & 3,3216 & 3,0441 & $(*)$ \\
Turismo de segunda residencia & 3,0609 & 3,6018 & 2,9378 & 3,5294 & $(*)$ \\
“Turismo de marcha” & 2,5136 & 2,7389 & 2,4090 & 3,3015 & $(*)$ \\
All inclusive & 2,3609 & 2,2832 & 2,4123 & 1,9191 & $(*)$ \\
\hline
\end{tabular}

(*) Diferencia entre islas significativa al 0,01 según la prueba F de Snedecor.

Fuente: elaboración propia.

- El turismo basado en la cultura y el patrimonio permite aumentar el orgullo de pertenencia de los residentes a una comunidad local. La cultura y el patrimonio era un elemento considerado por muchos como infrautilizado en la oferta turística. Aunque también había opiniones que consideraban que el volumen de turistas que atraería sería pequeño y en todo caso sólo serviría para complementar la oferta actual. El turismo basado en la cultural y el patrimonio era el mejor valorado en las islas $(4,1570)$, después del turismo de naturaleza y el turismo rural. El motivo de esta valoración tan positiva es el mismo que en el caso del turismo basado en la naturaleza pero aplicado al entorno humano. A muchos residentes les gustaba que su cultura y su patrimonio fueran motivo de admiración y, consecuentemente, de visitas turísticas.

- El turismo de congresos, seminarios y ferias es un clásico de los destinos urbanos que desde hace algunos años plantean como herramienta de desestacionalización en algunos destinos tradicionales de sol y playa, como es el caso de Baleares. Los congresos, seminarios y ferias tenían un muy buen grado de aceptación en Baleares 
(4,0945), no existiendo diferencias significativas entre islas. Se trata de un tipo de turismo poco significativo en las islas pero bastante atractivo para la ciudadanía en general y las autoridades en particular.

- El turismo de sol y playa familiar era el tradicional en Baleares y el más típico en gran parte de la costa mediterránea. Esta importancia tradicional genera unos pros y contras, pero la valoración general era de aceptación (3,7058). Por islas, destacaba Ibiza $(4,1416)$ por tener la valoración más positiva hacia este tipo de turismo. Probablemente, el elevado grado de aceptación se deba a que no es el mejor pero si el único que podía mantener los volúmenes de turistas y por tanto generar suficientes ingresos para la población.

- El cicloturismo, entendido como la llegada de ciclistas amateurs o profesionales a la isla para entrenar, es uno de los tipos de turismo alternativo que se suelen considerar como herramienta de desestacionalización turística. El cicloturismo era aceptable en el conjunto de las islas $(3,6188)$, pero sobretodo en Ibiza $(3,9867)$. Siendo Mallorca la que mostraba la puntuación más moderada $(3,5586)$. El cicloturismo pertenece a un grupo de productos turísticos con demanda cuantitativa pequeña pero con la característica de permitir atraer turismo en las temporadas media y baja. Desde hace años se han desarrollado rutas ciclistas con la finalidad de fomentar y facilitar las excursiones ciclistas como complemento a la oferta existente.

- A parte de por el cicloturismo, en la encuesta se preguntó por otros tipos de turismo deportivo (turismo deportivo en general). Las ofertas deportivas en general mostraban un mayor grado de aceptación que el cicloturismo $(3,9509$ frente a 3,6188). Seguía siendo Ibiza la que mostraba mayor grado de aceptación de este tipo de turismo $(4,1858)$, pero donde había mayor mejoría en la puntuación en relación al cicloturismo era en Mallorca (3,9107 frente al 3,5586 del cicloturismo) y Menorca (4,0074 frente al 3,6765 del cicloturismo).

- El turismo náutico tiene gran importancia en destinos de costa. Este tipo de turismo se caracteriza por tener un elevado poder adquisitivo y generar pocos problemas. Aunque competiciones como la Copa del Rey de Vela celebrada todos los años en Palma de Mallorca son un importante atractivo para este tipo de turismo, otros destinos de moda, como es el caso de Ibiza, consiguen atraer una gran cantidad de turistas que tienen como parte significativa de su estancia la navegación. El turismo 
náutico tiene como puntos positivos que es turismo con alto o muy alto poder adquisitivo, no es conflictivo y no implica una ocupación del territorio. Entre las desventajas hay: el impacto ambiental de los clubs náuticos necesarios para dar refugio a las embarcaciones, la contaminación del agua por vertidos ocasionados por las embarcaciones, los daños en el fondo marino provocados por cadenas y anclas, y el impacto visual que genera en algunos casos la enorme aglomeración de embarcaciones en una misma zona. El turismo náutico poseía un grado de aceptación moderado en Mallorca $(3,4540)$ y mucho mejor en Ibiza $(4,1106)$ y en Menorca $(3,9853)$. El turismo náutico tiene la peculiaridad de que no genera efectos más allá de una estrecha franja de costa. Mallorca es la isla más grande de Baleares y posee bastante territorio y población demasiado alejada de la costa como para obtener beneficios del turismo náutico y los puertos deportivos.

- Las casas y apartamentos vacacionales en alquiler tenían un nivel de aceptación moderado en las islas $(3,5195)$, en especial en Mallorca $(3,4242)$. Se trata de una oferta turística que, a pesar de la aceptación mayoritaria, se encontraba en la parte baja de la lista de preferencias, no resultando del todo convincente para los residentes. La posible explicación habría que buscarla en el hecho de que los turistas que alquilan casas no son considerados malos turistas pero no son los que dejan más ingresos. Además las viviendas en alquiler generan menos empleo que los hoteles, siendo considerado por algunos expertos mejor el desarrollo basado en hoteles (CHÍAS, 2005).

- Los campos de golf es un producto turístico que ha traído polémica en la prensa desde hace años. Los detractores alegan impactos medioambientales y los partidarios alegan que el golf permite atraer turismo de calidad y que para regar el césped se usaría agua depurada. En realidad el principal escollo que plantean los críticos es la tendencia a construir grandes urbanizaciones residenciales alrededor de los campos de golf. Por tanto, a priori la postura hacia los campos de golf parece más dividida y politizada que en otros productos turísticos. Los campos de golf poseían una moderada aceptación en el conjunto de Baleares $(3,3182)$. Por islas, Menorca $(3,0441)$ tenía una postura general de indiferencia y Mallorca $(3,3216)$ e Ibiza $(3,4602)$ de moderada aceptación.

- El turismo de segunda residencia, es decir personas no residentes propietarias de viviendas que usan sólo durante sus vacaciones. El turismo de segunda residencia tenía una valoración general ambigua $(3,0609)$, ya que las valoraciones positivas y las 
negativas se compensaban. Por islas había una postura ligeramente negativa en Mallorca $(2,9378)$ y moderadamente positiva en Ibiza $(3,6018)$ y Menorca $(3,5294)$. Es un tipo de oferta menos valorada que las casas en alquiler y en Mallorca creó suspicacias con la masiva compra de viviendas por parte de alemanes en los noventa. El turismo de segunda residencia es, al igual que las casas vacacionales en alquiler, un tipo de turismo que necesita la urbanización de una gran cantidad de territorio pero genera pocos puestos de trabajo una vez construidas las viviendas.

- El "turismo de marcha" (discotecas, bares, etc.), o turismo clubber. La postura mayoritaria en Baleares hacia el turismo basado en el ocio nocturno era de no aceptación (2,5136). La única isla que tenía una postura de moderada aceptación era Menorca (3,3015), probablemente porque en esa isla el sector está poco desarrollado. Los inconvenientes de este tipo de turismo (ruidos, suciedad, problemas de tráfico, etc.) junto con la imagen de turismo de baja calidad son los motivos más probables del rechazo a este tipo de turismo.

- La oferta de "todo incluido" o All inclusive está implantada en establecimientos de algunas zonas muy turísticas de las islas. Esta oferta fue copiada de los Resort situados en destinos tropicales, en los cuales era necesario ofrecer este tipo de oferta, y posteriormente importada en destinos más tradicionales. El "todo incluido" tiene la parte positiva para el cliente de permitir conocer y controlar de antemano el gasto que se realizará y para el empresario de retener al turista y su gasto en el establecimiento. La parte negativa es que la oferta complementaria del destino (tiendas, restaurantes, bares, etc.) sufre un durísimo recorte en sus ingresos, ya que los consumos que realizan los turistas en el hotel los pierden ellos. En destinos turísticos tradicionales, como es el caso de Baleares, la gran presencia de oferta complementaria convierte en nada popular el "todo incluido" (2,3609 de valoración global). Esta oferta era mal vista en todas las islas. Donde era considerada más inaceptable era en Menorca $(1,9191)$ y donde era menos rechazada era en Mallorca $(2,4123)$.

Es posible afirmar que los residentes consideraban que había un conjunto de productos turísticos muy aceptables (turismo rural y de naturaleza, turismo cultural, congresos y ferias, y turismo deportivo), con puntuaciones entre 4,28 y 3,95, otros productos bastante aceptables (turismo de sol y playa, cicloturismo, turismo náutico, y casas en alquiler), productos moderadamente aceptables (golf) o sin una postura clara a 
favor o en contra (segundas residencias), y productos con problemas de aceptación (clubbers y all inclusive). Dentro del primer grupo había ofertas relativamente nuevas, que permiten explotar atractivos infrautilizados y desestacionalizar la temporada turística, aunque son cuantitativamente poco significativas. En el segundo grupo hay ofertas con valoración favorable pero con algún problema detectado por parte de los residentes. Finalmente, había cuatro ofertas (golf, segundas residencias, turismo clubber y all inclusive) rodeadas de fuertes polémicas en la opinión pública insular.

\subsection{SEGMENTACIÓN SEGÚN VALORACIÓN DE LAS OFERTAS TURÍSTICAS}

Como parte del análisis de los productos y ofertas turísticas se procedió a una segmentación de la muestra. Esta segmentación se realizó mediante un Análisis Clúster (Algoritmo de Howard-Harris), usando el programa DYANE 4.0 (SANTESMASES, 2009), conservando la segmentación mayor que cumplía el criterio de que en todos los grupos haya al menos un $10 \%$ de la muestra y una nueva división no permitiera explicar más del 5\% de la varianza. Los cuatro grupos resultantes son (Cuadro 3):

Los "partidarios" $(20,0 \%)$. Valoraban de forma muy positiva todas las ofertas planteadas, excepto el All inclusive, que poseía una valoración entre ambigua y negativa. Era el grupo con las puntuaciones más altas en todos los productos, el 30,94\% poseía estudios superiores y el $24,63 \%$ trabajaba en el sector turístico o en contacto con los turistas. Era un grupo poco importante en Mallorca $(16,08 \%)$, pero bastante importante en Ibiza $(33,63 \%)$ y muy importante en Menorca (41,18\%). Eran los más entusiastas con el turismo, a excepción de la oferta del "todo incluido".

CUADRO 3 - VALORACIÓN DE LAS OFERTAS POR LOS SEGMENTOS GENERADOS.

\begin{tabular}{lccccc}
\hline & $\begin{array}{c}\text { Baleares } \\
\mathbf{n = 1 8 7 3}\end{array}$ & $\begin{array}{c}\text { Partidarios } \\
\mathbf{n = 3 7 5}\end{array}$ & $\begin{array}{c}\text { Anti-clubber } \\
\mathbf{n = 5 9 5}\end{array}$ & $\begin{array}{c}\text { Moderados } \\
\mathbf{n = 4 6 7}\end{array}$ & $\begin{array}{c}\text { Críticos } \\
\mathbf{n = 4 3 6}\end{array}$ \\
\hline Turismo Rural & 4,277 & 4,707 & 4,509 & 3,475 & 4,447 \\
Turísticos de naturaleza & 4,247 & 4,669 & 4,503 & 3,383 & 4,461 \\
Turismo cultura & 4,157 & 4,520 & 4,383 & 3,379 & 4,369 \\
Congresos, Ferias, etc. & 4,095 & 4,552 & 4,377 & 3,394 & 4,067 \\
Otro turismo deportivo & 3,951 & 4,472 & 4,207 & 3,281 & 3,872 \\
Turismo de sol y playa & 3,706 & 4,459 & 3,656 & 3,379 & 3,477 \\
Cicloturismo & 3,619 & 4,197 & 4,032 & 2,861 & 3,369 \\
Turismo Náutico & 3,572 & 4,461 & 4,092 & 3,263 & 2,427 \\
Casas en alquiler & 3,520 & 4,299 & 3,556 & 3,184 & 3,158 \\
Golf & 3,318 & 4,157 & 3,795 & 3,193 & 2,080 \\
Turismo 2 ${ }^{\text {a residencia }}$ & 3,061 & 4,093 & 3,212 & 2,809 & 2,236 \\
"Turismo de marcha" & 2,514 & 3,979 & 1,852 & 2,726 & 1,929 \\
All inclusive & 2,361 & 2,917 & 2,005 & 2,884 & 1,807 \\
\hline
\end{tabular}

Fuente: elaboración propia. 
- Los "Anti-clubber" (31,8\%). Era el segundo grupo, después de los "Partidarios", con puntuaciones más elevadas (superiores a la media de la muestra), salvo en el caso del All inclusive con una fuerte oposición $(2,005)$ sólo superada por los críticos $(1,807)$. Este grupo se caracterizaba por ser el más contrario al turismo clubber $(1,852)$, hecho que destacaba con los elevados niveles de apoyo a la mayoría de productos. Era uno de los grupos con mayor nivel de estudios (el 38,79\% poseía estudios superiores) y el grupo con mayor contacto con los turistas (el 32,61\% trabajaba en el turismo o en contacto con turistas). Eran menos abundantes en Menorca (24,26\%) y bastante abundantes en Ibiza (37,61\%). Eran muy partidarios de las diversas ofertas de turismo con la excepción del all inclusive y el turismo clubber.

- Los "Moderados" (24,9\%). Este grupo se caracterizaba por puntuaciones moderadas: consideraban moderadamente inaceptable el All inclusive, el cicloturismo, el turismo clubber y el turismo de segunda residencia (puntuaciones entre 2,72 y 2,88), y el resto de ofertas eran consideradas moderadamente aceptables (puntuaciones inferiores a 3,48). Era un grupo con bajo contacto con los turistas (el 21,92\% trabajaba en el sector o en contacto con los turistas), menor nivel de estudios que otros grupos (el $31,74 \%$ poseía estudios superiores) y poca presencia en Ibiza $(16,37 \%)$ y Menorca $(16,91 \%)$. Era un grupo con menos estudios que la media y con poco contacto con el sector turístico, lo cual lleva a que mostraran respuestas moderadas, consecuencia de disponer de menor información directa que otros grupos.

- Los "Críticos" (23,3\%). Valoraban por encima de la media algunas ofertas (turismo rural, turismo de naturaleza y turismo cultural), valoraban poco otras ofertas (turismo de sol y playa, casas vacacionales y cicloturismo), se oponían de forma muy intensa al All inclusive y el turismo clubber, y mostraban una importante oposición a lo que podría denominarse el "turismo de ricos" (golf, turismo náutico y turismo de segunda residencia). Era el grupo con menor contacto con el turismo (el 20,84\% de la muestra trabajaba en el turismo o en contacto con los turistas) y mayor nivel de estudios (el $43,65 \%$ poseía estudios superiores). Eran pocos en Menorca $(17,65 \%)$ y escasos en Ibiza (12,39\%). 
CUADRO 4 - DISTRIBUCIÓN DE LOS SEGMENTOS POR ISLA.

\begin{tabular}{lcccc}
\hline & Baleares & Ibiza & Mallorca & Menorca \\
\hline Partidarios & $20,02 \%$ & $33,63 \%$ & $16,08 \%$ & $41,18 \%$ \\
Anti-clubber & $31,77 \%$ & $37,61 \%$ & $31,57 \%$ & $24,26 \%$ \\
Moderados & $24,93 \%$ & $16,37 \%$ & $26,94 \%$ & $16,91 \%$ \\
Críticos & $23,28 \%$ & $12,39 \%$ & $25,41 \%$ & $17,65 \%$ \\
\hline
\end{tabular}

Fuente: elaboración propia.

Comparando con la media del archipiélago (Cuadro 4), se observa que Mallorca poseía más "Moderados" y "Críticos" que las otras dos islas. Mallorca es una isla mucho más grande que Menorca e Ibiza y ello facilita la existencia de zonas, sectores e individuos que no poseen una vinculación muy intensa con la actividad turística. Estos individuos con poco contacto con el turismo pueden mostrar respuestas moderadas, formando el grupo de los "Moderados", o poseer respuestas contundentes, tanto positivas (turismo rural, de naturaleza o cultural) como negativas (All inclusive, turismo clubber, golf, turismo náutico y turismo de segunda residencia), hacia los diversos tipos de ofertas propuestos, formando el grupo de los "Críticos". Ibiza destacaba por la presencia de "Partidarios" y "Anti-clubbers" por la fuerte dependencia del turismo que posee la isla y la enorme importancia de las macrodiscotecas en la oferta de la isla, que hacen patentes los inconvenientes de esta oferta. En Menorca destacaba el grupo de los "Partidarios", posiblemente porque algunas de las ofertas más polémicas (por ejemplo el ocio nocturno) son poco representativas en esta isla.

\section{CONCLUSIONES}

Las ofertas más positivamente valoradas eran el turismo rural y el basado en la naturaleza, seguidas de cerca por el turismo basado en la cultura y el patrimonio. Las viviendas en alquiler, las segundas residencias y los campos de golf tenían una valoración general positiva pero muy baja y el "todo incluido" y el turismo de ocio nocturno poseían valoraciones generales negativas.

El "todo incluido" poseía elevados grados de falta de aceptación en todas las islas y segmentos analizados debido al perjuicio económico que representa para la oferta complementaria y la falta de contacto de los turistas con el entorno de la isla. 
Al comparar las diversas islas se observaba que a mayor dependencia económica del turismo (Ibiza y Menorca) mayor grado de apoyo a las diversas ofertas turísticas. Mallorca tenía la valoración más baja en el promedio general y en 10 de los 13 elementos analizados. Si se tiene en cuenta que la muestra de Mallorca poseía un $35,61 \%$ de personas que trabajaban en el sector turístico, mientras que Ibiza tenía el $53,85 \%$ y Menorca el 67,18\%. La explicación es que los individuos con un conocimiento indirecto del turismo suelen tener valoraciones más moderadas que los individuos en contacto con el turismo. Ibiza tenía la valoración más alta de las tres islas en el promedio general y en 9 de los 13 elementos analizados. Esto se explica por la mayor dependencia turística de Ibiza, lo cual hace que las personas tengan mayor conciencia de su importancia, aunque no trabajen directamente en el sector.

En general, se observa que las ofertas turísticas que generan menor impacto en la comunidad residente e implican un mayor interés de los turistas por la sociedad y el entorno local poseían mayor grado de aceptación, mientras que la oferta turística con más impactos negativos y que implica menor interacción con los residentes poseía bajos niveles de aceptación por parte de los residentes. Es posible afirmar que un turismo de tipo Nómada (LANQUAR, 2001), “extremo impulsor” (BIGNÉ; FONT; ANDREU, 2000) o alocéntricos (PLOG, 1974) es la opción más deseable para los residentes y, por tanto, para el desarrollo de un destino.

\section{REFERENCIAS}

AGÈNCIA DE TURISME DE LES ILLES BALEARS. El turisme a les Illes Balears. Anuari 2012. Palma de Mallorca: Agència de Turisme de les Illes Balears, 2013.

AGUILÓ, E.; ROSSELlÓ, J. Host community perceptions. A cluster análisis. Annals of Tourism Research, v. 32, n. 4, p. 925-941, 2005.

AP, J.; CROMPTON, J. L. Developing and testing a tourism impact scale. Journal of Travel Research, v. 37, n. 2, p. 120-130, 1998.

BESCULIDES, A.; LEE, M.; MCCORMICK, P. Resident's perceptions of the cultural benefits of tourism. Annals of Tourism Research, v. 29, n. 2, p. 303-319, 2002.

BIGNÉ, J. E.; FONT, X.; ANDREU, L. Marketing de destinos turísticos. Análisis y estrategias de desarrollo. Madrid: Editorial ESIC, 2000. 
BRIDA, G. G.; OSTI, L.; BARQUET, A. Segmenting resident perceptions towards tourism - A cluster analysis with a multinomial logit model of a mountain community. International Journal of Tourism Research, v. 12, n. 5, p. 591-602, 2010.

BROWN, D.; ROSEMAN, M. G.; HAM, S. Perceptions of a Bible Belt State's proposed casino gaming legislation bi religious affiliation: the case of Kentucky residents. UNLV Gaming Research \& Review Journal, v. 7, n. 1, p. 49-58, 2003.

BRUCKNER, P.; FINKIELKRAUT, A. Au Coin de la Rue. L'aventure. Paris: Èditions du Seuil, 1979.

CHÍAS, J. El negocio de la felicidad. Desarrollo y marketing turístico de países, regiones, ciudades y lugares. Madrid: Prentice Hall-Pearson Educación, 2005.

FISCHER, H. Warum Samoa? Touristen und Tourismus in der Südsee. Berlin: Reimer, 1984.

FREDLINE, E.; FAULKNER, B. Host community reactions: A cluster analysis. Annals of Tourism Research, v. 27, n. 3, p. 763-784, 2000.

GOFFMAN, E. Encounters: Two Studies in the Sociology of Interaction. Indianapolis: Bobbs-Merrill, 1961.

GÓMEZ, L.; SAN MARTÍN, J.; BERTICHE, C. A model of tourism experience and attitude change. Annals of Tourism Research, v. 26, n. 4, p. 1024-1027, 1999.

GURSOY, D.; JUROWSKI, C.; UYSAL, M. Resident attitudes: a structural Modeling Approach. Annals of Tourism Research, v. 29, n. 1, p. 79-105, 2002.

GURSOY, D.; KENDALL, K. W. Hosting mega events: modeling locals' support. Annals of Tourism Research, v. 33, n. 3, p. 603-623, 2006.

GURSOY, D.; RUTHERFORD, D. Host atttitudes toward tourism: An Improved Structtural Model. Annals of Tourism Research, v. 31, n. 3, p. 495-516, 2004.

HARALAMBOPOULOS, N.; PIZAM, A. Perceived impacts of tourism: the case of Samos. Annals of Tourism Research, v. 23, n. 3, p. 503-526, 1996.

HENNIG, C. Touristenbeschimpfung. Zur geschichte des anti-tourismus. Zeitschrift für Volkskunde, v. 93, n. 1, p. 31-41, 1997.

IBESTAT. INSTITUT BALEAR D'ESTADÍSTICA. 2012. Disponible en: <http://ibestat.caib.es>. Acceso en: 20/11/2013.

JACOBSEN, J. K. S. Anti-tourist attitudes: mediterranean charter tourism. Annals of Tourism Research, v. 27, n. 2, p. 284-300, 2000. 
JANES, P.; COLLISON, J. Community leader perceptions of the social and economic impacts of indian gaming. UNLV Gaming Research \& Review Journal, v. 8, n. 1, p. 13-30, 2004.

JUROWSKI, C.; GURSOY, D. Distance effects on residents' attitudes toward tourism. Annals of Tourism Research, v. 31, n. 2, p. 296-312, 2004.

JUROWSKI, C.; UYSAL, M.; WILLIAMS, R. D. A theoretical analysis of host community resident reactions to tourism. Journal of Travel Research, v. 36, n. 2, p. 3$11,1997$.

KIM, S. S.; PETRICK, J. F. Residents' perceptions on impacts of the FIFA 2002 World Cup: the case of Seoul as a host city. Tourism Management, v. 26, n. 1, p. 25-38, 2005.

LANQUAR, R. Marketing turístico: de lo global a lo local. Barcelona: Editorial Ariel, 2001.

LEE, C. K.; KIM, S. S.; KANG, S. Perceptions of casino impacts: A Korean longitudinal study. Tourism Management, v. 24, n. 1, p. 45-55, 2003.

MADDOX, R. N. Measuring satisfaction with tourism. Journal of Travel Research, v. 23, n. 3, p. 2-5, 1985.

MASON, P.; CHEYNE, J. Resident's attitudes to proposed tourism development. Annals of Tourism Research, v. 27, n. 2, p. 391-411, 2000.

MILMAN, A.; PIZAM, A. Social impact of tourism on Central Florida. Annals of Tourism Research, v. 15, n. 2, p. 191-204, 1988.

MURPHY, P. E. Tourism: A community approach. New York: Routledge, 1985.

PEARCE, D. G. Tourist organizations in Sweden. Tourism Management, v. 17, n. 6, p. 413-424, 1996.

PEARCE, P. L. Tourists and their Hosts: Some Sociological and Psychological Effects of International Contact. In: BOCHNER, S. (edi.). Cultures in Contact. Oxford: Pergamon, 1982. p. 199-221.

PLOG, S. C. Why destination areas rise and fall in popularity. Cornell Hotel and Restaurant Administration Quarterly, v. 14, n. 4, p. 55-58, 1974.

RITCHIE, J. R. B. Consensus policy formulation in tourism: Measuring resident views via survey research. Tourism Management, v. 9, n. 3, p. 199-212, 1988.

SANTESMASES, M. DYANE Versión 4: Diseño y análisis de encuestas en investigación social y de mercados. Madrid: Ediciones Pirámide, 2009. 
SCHEUCH, E. K. Ferien und tourismus als neue formen der freizeit. In: SCHEUCH, E. K.; MEYERSOHN, R. (edi.). Soziologie der Freizeit. Köln: Kiepenheuer \& Witsch, 1972. p. 304- 317.

TEYE, V.; SIRAKAYA, E.; SÖNMEZ, S. F. Resident's attitudes toward tourism development. Annals of Tourism Research, v. 29, n. 3, p. 668-688, 2002.

URBAIN, J. D. L'Idiot du Voyage. Paris: Plon, 1991.

VONG, F. Changes in residents' gambling attitudes and perceived impacts at the fifth anniversary of Macao's gaming deregulation. Journal of Travel Research, v. 47, n. 3, p. 388-397, 2009.

VONG, F.; MCCARTNEY, G. Mapping resident perceptions of gaming impact. Journal of Travel Research, v. 44, n. 2, p. 177-187, 2005.

WEAVER, D. B.; LAWTON, L. J. Resident perceptions in the urban-rural frige. Annals of Tourism Research, v. 28, n. 2, p. 439-458, 2001.

WILLIAMS, J.; LAWSON, R. Community issues and resident opinions of tourism. Annals of Tourism Research, v. 28, n. 2, p. 269-290, 2001.

Recebido em: 05-09-2014.

Aprovado em: 05-10-2014. 